\title{
Time Dependent Entropy and Decoherence in a Modified Quantum Damped Harmonic Oscillator
}

\author{
F. B. Pelap ${ }^{1 *}$, A. Fomethe ${ }^{1}$, A. J. Fotue ${ }^{2}$, M. P. Djemmo Tabue ${ }^{1,2}$ \\ ${ }^{1}$ Laboratoire de Mécanique et de Modélisation des Systèmes Physiques (L2MSP), Faculté des Sciences, \\ Université de Dschang, Dschang, Cameroon \\ ${ }^{2}$ Laboratory of Mesoscopy and Multilayers Structures, Faculty of Science, University of Dschang, Dschang, \\ Cameroon \\ Email: fbpelap@yahoo.fr
}

Received 17 November 2014; revised 2 December 2014; accepted 9 December 2014

Copyright @ 2014 by authors and Scientific Research Publishing Inc.

This work is licensed under the Creative Commons Attribution International License (CC BY). http://creativecommons.org/licenses/by/4.0/

(c) (1) 0pen Access

\section{Abstract}

The time dependence of probability and Shannon entropy of a modified damped harmonic oscillator is studied by using single and double Gaussian wave functions through the Feynman path method. We establish that the damped coefficient as well as the system frequency and the distance separating two consecutive waves of the initial double Gaussian function influences the coherence of the system and can be used to control its decoherence.

\section{Keywords}

Modified Damped Harmonic Oscillator, Feynman Path Integral, Decoherence, Shannon Entropy, Distribution Probability

\section{Introduction}

The study of dissipative systems and their quantization is of a great theoretical and practical value in view of many different situations in which dissipative phenomena with a quantum origin manifest themselves [1]. Within these dissipative systems, the damped harmonic oscillator (DHO) is the simplest quantum systems displaying the dissipation of energy. It is of great physical importance and has found many applications especially in quantum optics [2] [3]. For example, it plays a central role in the quantum theory of lasers and masers [4]. Moreover, damped harmonic oscillators are used to investigate the quantum decoherence (QD) phenomenon whose role

*Corresponding author.

How to cite this paper: Pelap, F.B., Fomethe, A., Fotue, A.J. and Djemmo Tabue, M.P. (2014) Time Dependent Entropy and Decoherence in a Modified Quantum Damped Harmonic Oscillator. Journal of Quantum Information Science, 4, 214-226. 
became relevant in many interesting physical problems such as quantum computation and quantum information processing [5], material science [6]-[8], heavy ion collisions [9], quantum gravity and cosmology [10]-[22], and condensed matter physics [23]-[25]. In many cases, physicists are interested in understanding the causes of QD in order to prevent decoherence from damaging quantum states and protect the information stored in these states. Thus, decoherence is responsible for washing out the quantum interference effects which are desirable to be seen as signals in some experiments. However, QD has negative effects in many areas such as quantum computation and quantum control of atomic and molecular processes. The physics of information and computation is a domain where decoherence is an obvious major obstacle in the implementation of information-processing hardware. It takes advantage on the superposition principle [26]. QD is a condition that has to be satisfied in order that a system could be considered as classical. This condition requires that the system should be in one of relatively permanent states (called by Zurek "preferred states") and the interference between different states should be negligible [27] [28]. The loss of coherence can be achieved by introducing an interaction between the system and environment [23] [29] [30].

Nowadays, a great deal of research is dedicated to understanding decoherence in harmonic oscillator [31] [32]. Isar et al. [33] determine the degree of quantum decoherence of a harmonic oscillator interacting with a thermal bath using Lindblad theory [34] [35]. Other authors [36] use a semi-classical approach to examine decoherence in a harmonic oscillator coupled to a thermal harmonic bath. Darius et al. [37] exploit the Feynman path integral to study the memory in a non-locally damped oscillator. Moreover, Ozgur et al. [32] determine the time dependence of Leipnik's entropy in the damped harmonic oscillator via path integral techniques. Another strategy to describe dissipative quantum systems is based on the idea of Bateman [38].

In this paper, we investigate the coherence of the damped harmonic oscillator using the Caldirola-Kanai model [39] but based on the idea of Bateman [38]. This model is known as a popular model used to describe dissipative systems coupled to a harmonic bath. It has many applications like reproducing classical effects or giving a good Hamiltonian necessary to exhibit the phenomenon of decoherence [38]. This paper is organized as follows. In Section 2, we present the mathematical tools based on the path integral formalism. We also discuss the case of the damped harmonic oscillator and build the associated propagator. In Section 3, we derive the general expressions of the thermodynamic parameters of the system, and analyze the effects of the damping constant on the distribution probability and the Shannon entropy for a single Gaussian wave function. The influence of the system's frequency has also been measured on these parameters. Hence, we consider a double Gaussian wave function and make numerical investigations appreciate the impact of the damping constant, the distance separating the two wave functions and the system's frequency on the thermodynamic characteristics of the system. Discussion and concluding remarks are given in the last section.

\section{Fundamental Definitions}

We start by presenting the model which consists of a particle of mass $m$, labeled by the position variable $q$ and the momentum $p$. Then follows the description of the used mathematical tools which is the path integral formalism introduced by Feynman [40]. These tools suggest that the transformation function called propagator is analogue to $\exp \left(\frac{i}{\hbar} S_{c l}\right)$ in which $S_{c l}$ stands for the action, solution of Hamilton-Jacobi equation. On the other way, the transition amplitude of the particle (of mass $\mathrm{m}$ ) from the position $q_{a}$ at time $t_{a}$ to the position $q_{b}$ at time $t_{b}$, known as the propagator, represents the solution of the Schrodinger equation. Nowadays, several problems of physics are solved via these techniques [32] [41].

Next, we consider the Bateman Hamiltonian [38] defined as:

$$
H=\bar{p} p-\gamma[x \bar{p}-\bar{x} p]+\Omega^{2} \bar{x} x
$$

where $\bar{p}$ and $\bar{x}$ are the mirror variables corresponding to the coordinate $x$ and the momentum $p$. The quantities $\gamma$ and $\Omega$ are respectively the damped coefficient and the system frequency. The associated lagrangian is given by

$$
L=\dot{\bar{x}} \dot{x}-x \bar{x}+\gamma(x \dot{\bar{x}}-x \bar{x})
$$

Using Euler-Lagrange equation, we derive the following two motion equations [42]: 


$$
\left\{\begin{array}{l}
\ddot{x}+\gamma \dot{x}+\Omega^{2} x=0 \\
\ddot{\bar{x}}+\gamma \dot{\bar{x}}+\Omega^{2} \bar{x}=0
\end{array}\right.
$$

Bateman's dual Hamiltonian describes classical mechanics correctly, but this model faces some difficulties. It violates Heisenberg's principle for $\gamma \neq 0$. Therefore, to solve quantum mechanical problem, Caldirola-Kanai [39] build a theory based on the idea of Bateman dissipative system by considering the standard Hamiltonian of harmonic oscillator with time dependent mass given by [39] $m(t)=m_{0} \exp (2 \gamma t)$. Hence, the Hamiltonian and the Lagrangian of the system become respectively:

$$
\begin{aligned}
& H=\frac{p^{2}}{2 m(t)}+\frac{1}{2} m(t) \omega^{2} x^{2}, \\
& L=p \dot{x}-H=\exp (2 \gamma t)\left[\frac{1}{2} m \dot{x}^{2}-\frac{1}{2} m \omega^{2} x^{2}\right] .
\end{aligned}
$$

Here, $\omega$ is the system frequency. From the Lagrangian theory and exploiting quantities (4), the equation of motion takes the form:

$$
\ddot{x}+2 \gamma \dot{x}+\Omega^{2} x=0
$$

The classical solution of (5) is given by

$$
x(t)=C_{1} \exp \left(a_{1} t\right)+C_{2} \exp \left(a_{2} t\right)
$$

where in $a_{1}$ and $a_{2}$ are complex quantities defined as: $a_{1}=-\gamma-i \Omega, a_{2}=-\gamma+i \Omega$ with $\Omega=\sqrt{\omega^{2}-\gamma^{2}}$. The integration constants $C_{1}$ and $C_{2}$ are evaluated when the particle moves from the position $x_{a}$ at the time $t_{a}$ to the position $x_{b}$ at time $t_{b}$. The determination of the propagator is convenient for founding quantum mechanical solution for this Hamiltonian. Therefore, the classical action $S_{c l}$ is defined as:

$$
S_{c l}=\int L(x, \dot{x}, t) \mathrm{d} t=\int_{t_{a}}^{t_{b}} \frac{m}{2}\left(\dot{x}^{2}-\omega^{2} x^{2}\right) \mathrm{d} t=\frac{m_{0}}{2} \int_{t_{a}}^{t_{b}}\left(\dot{x}^{2}-\omega^{2} x^{2}\right) \mathrm{d} t
$$

whose computation for the current study case leads to:

$$
S_{c l}=\frac{m_{0} \Omega \mu_{1}}{\exp \left\{-2 \gamma\left(t_{a}+t_{b}\right)\right\} \sin \left\{\Omega\left(t_{a}-t_{b}\right)\right\}}+\frac{m_{0} \gamma \mu_{2}}{\exp \left\{-2 \gamma\left(t_{a}+t_{b}\right)\right\}}
$$

in which we set:

$$
\begin{aligned}
& \mu_{1}=\left[x_{b}^{2} \exp \left(-2 \gamma t_{a}\right)+x_{a}^{2} \exp \left(-2 \gamma t_{b}\right)\right]-2 x_{a} x_{b} \exp \left[-\gamma\left(t_{a}+t_{b}\right)\right]+\cos \left\{\Omega\left(t_{a}-t_{b}\right)\right\}, \\
& \mu_{2}=x_{b}^{2} \exp \left(-2 \gamma t_{a}\right)-x_{a}^{2} \exp \left(-2 \gamma t_{b}\right) .
\end{aligned}
$$

From the classical action, the expression of the corresponding propagator is defined below.

$$
\aleph\left(x, x^{\prime}, t\right)=\frac{m \Omega}{2 \pi i \hbar \sin (\Omega t)} \exp \left(\frac{i}{\hbar} S_{c l}\right)
$$

Substituting (7) into (8), we obtain the following expression for the quantum propagator of damped harmonic oscillator:

$$
\aleph\left(x_{a}, x_{b}, t\right)=\left[\frac{m_{0} \Omega}{2 \pi i \hbar \exp \left\{2 \gamma\left(t_{a}+t_{b}\right)\right\} \sin \left\{\Omega\left(t_{b}-t_{a}\right)\right\}}\right]^{1 / 2} \exp \left(\frac{i}{\hbar} S_{c l}\right)
$$

This result is identical to the one establish in [41] using the propagator method developed by Um et al. [43]. It also appears from (9) that the propagator $\aleph\left(x_{a}, x_{b}, t\right)$ depends on the damped coefficient $\gamma$ that links the system 
with the environment in which it evolves.

Hereafter, we intend to use the propagator (9) and derive some characteristic parameters (such as the distribution probability and the Shannon entropy) of the system subjected respectively to single and double Gaussian wave functions. These investigations aim to measure the impact of the environment on the behavior of the system when the latter progresses.

\section{Calculations and Results}

\subsection{System Properties under a Single Gaussian Wave Function}

In this section, we exploit the single Gaussian wave function to examine the impact of the environment on the distribution probability and the Shannon entropy for a specific damped harmonic oscillator and therefore, to measure its coherence.

\subsubsection{Distribution Probability}

Making use of [31], we determine the distribution probability for a single Gaussian wave packet to find the particle at coordinate $x$ at the time $t$. Nowadays, several problems of physics are solved via path integral techniques. It gives analytical solution for various coupling problems. This probability can be written in the Feynman-Hibbs form as [44]:

$$
P(x, t)=|\varphi(x, t)|^{2}=\int \mathrm{d} x_{b}^{\prime} \int \mathrm{d} x_{b} \aleph^{*}\left(x_{a}, x_{b}^{\prime}, t\right) \aleph\left(x_{a}, x_{b}, t\right) \varphi\left(x_{b}^{\prime}, 0\right) \varphi\left(x_{b}, 0\right)
$$

which presents the link between the distribution probability $P(x, t)$ and the propagator (9) for a given wave function $\varphi$. In expression (10), $\varphi\left(x_{b}, 0\right)$ designates the initial Gaussian wave packet centered at $x_{0}=0$ with [31]:

$$
\varphi\left(x_{b}, 0\right)=\left[2 \pi \sigma^{2}\right]^{-1 / 4} \exp \left(-\frac{x^{2}}{4 \sigma^{2}}\right)
$$

The quantity $\varphi\left(x_{b}^{\prime}, 0\right) \varphi^{*}\left(x_{b}, 0\right)$ defines the pure electronic density matrix and $\sigma^{2} \hbar / 2 m \Omega$. Based on (9), we evaluate the propagator

$$
\aleph^{*}\left(x_{a}, x_{b}^{\prime}, t\right) \aleph\left(x_{a}, x_{b}, t\right)=\left[\frac{m_{0} \Omega \exp \left(2 \gamma t_{b}\right)}{2 \pi \hbar \sin \left\{\Omega\left(t_{b}-t_{a}\right)\right\}}\right] \exp \left(\frac{i}{\hbar} S_{c l}\right)
$$

which takes the following form after substitution of the classical action $S_{c l}$ :

$$
\aleph^{*}\left(x_{a}, x_{b}^{\prime}, t\right) \aleph\left(x_{a}, x_{b}, t\right)=\left[\frac{m_{0} \Omega \exp \left(2 \gamma t_{b}\right)}{2 \pi \hbar \sin \left\{\Omega\left(t_{b}-t_{a}\right)\right\}}\right] \exp \left[a\left(x_{b}^{2}-x_{b}^{\prime 2}\right)+b\left(x_{b}-x_{b}^{\prime}\right)\right]
$$

With

$$
a=\frac{i}{\hbar}\left\{m_{0} \exp \left(2 \gamma t_{b}\right)\left[\Omega \cot \Omega\left(t_{b}-t_{a}\right)+\gamma\right]\right\}, \quad b=-\frac{2 i x_{a} m_{0} \Omega \exp \left[\gamma\left(t_{b}+t_{a}\right)\right]}{\hbar \sin \left[\Omega\left(t_{b}-t_{a}\right)\right]}
$$

Therefore, the distribution probability (10) yields the following expression:

$$
P=\frac{2 \sigma m_{0} \Omega \exp \left(2 \gamma t_{b}\right)}{\hbar \sin (\Omega t)}\left(\frac{1}{2 \pi}\right)^{1 / 2}\left\{\frac{1}{\sqrt{1-16 a^{2} \sigma^{4}}} \exp \left[\frac{2 b^{2} \sigma^{2}}{1-16 a^{2} \sigma^{4}}\right]\right\}
$$

This relation shows that the distribution probability is an explicit function of space and time. Figure 1(a) presents its evolution when the damping coefficient $\gamma$ is null (pure state). This plot shows that the probability decreases with space and increases with time. We observe that the probability behaves like in the case of free particle as shown in [32]. For non-zero values of $\gamma$ Figure 1(b) gives the evolution of the same probability. 


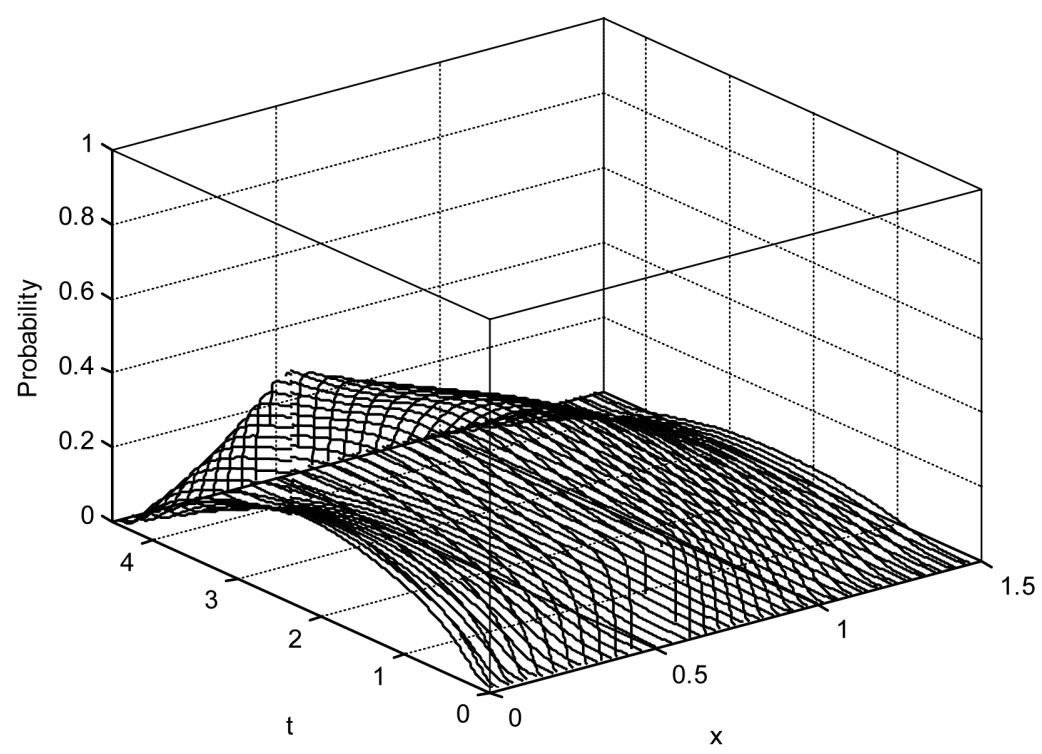

(a)

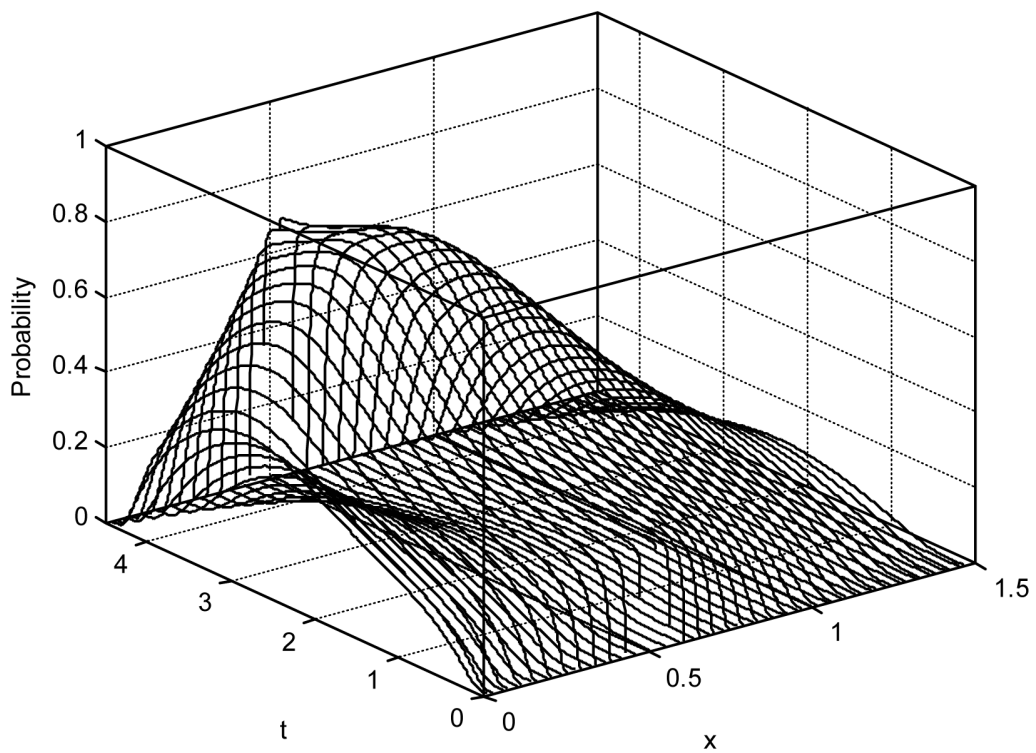

(b)

Figure 1. Distribution probability for a single Gaussian wave function with the parameters $\Omega=0.7, m_{0}=1, \hbar=1$ and two values of $\gamma$ (a): $\gamma=0$ (pure state) and (b): $\gamma=0.3$ (damped state).

From the obtained curve, one observes that the probability increases with the growth of the damping coefficient. This result traduces the fact that the interaction between the system and the environment induces a modification of the former. It also establishes the existence of the critical value $\gamma_{c}$ of $\gamma$ over which the probability will exceed unit. The determination of $\gamma_{c}$ is useless here since we are not concerned with the consequences of its existence in the present work. Furthermore, we intend to deeply examine another aspect of the coherence of the system by investigating the influence of the environment on the Shannon entropy.

\subsubsection{Shannon Entropy}

It is well known that the major way to appreciate the purity of a system is to study the evolution of its entropy. When this quantity tends to zero, we obtain a pure state. Decoherence stands for the loose of information in the 
system. This occurs when the exchange between the environment and the system affects the evolution of the concerned system. Mathematically, the entropy $S_{1}$ is defined by Boltzmann-Shannon as:

$$
S_{1}=-K_{B} \int_{-\infty}^{+\infty} P \ln P \mathrm{~d} x
$$

in which $K_{B}$ represents the Boltzmann constant and $P$ is the distribution probability defined by (14) for a single Gaussian function. Therefore, it appears that the Shannon entropy $S_{1}$ is an explicit function of time. But it also deals with the system frequency. Indeed, Figure 2 gives the evolution of this entropy in terms of time and system frequency for pure system. From this graph, we note that the entropy oscillates with time. Therefore, the information is periodically transferred between the environment and the system.

However, when $\gamma \neq 0$, Figure 3 presents the behavior of temporal evolution of the entropy versus the system

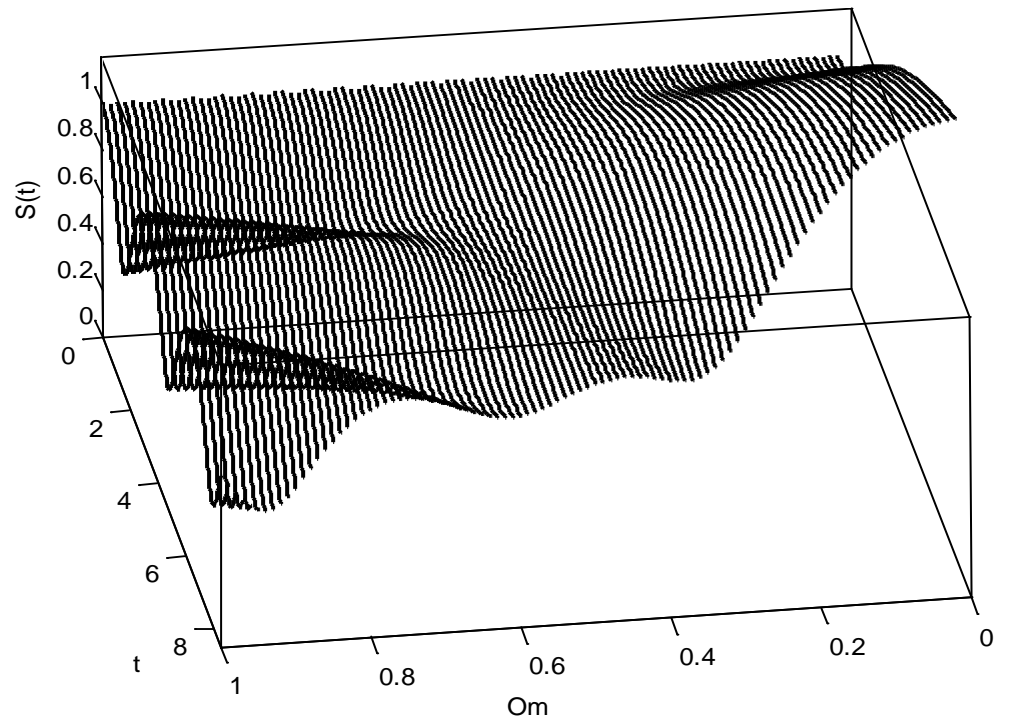

Figure 2. The 3D entropy for a simple harmonic oscillator with the parameters of Figure 1(a).

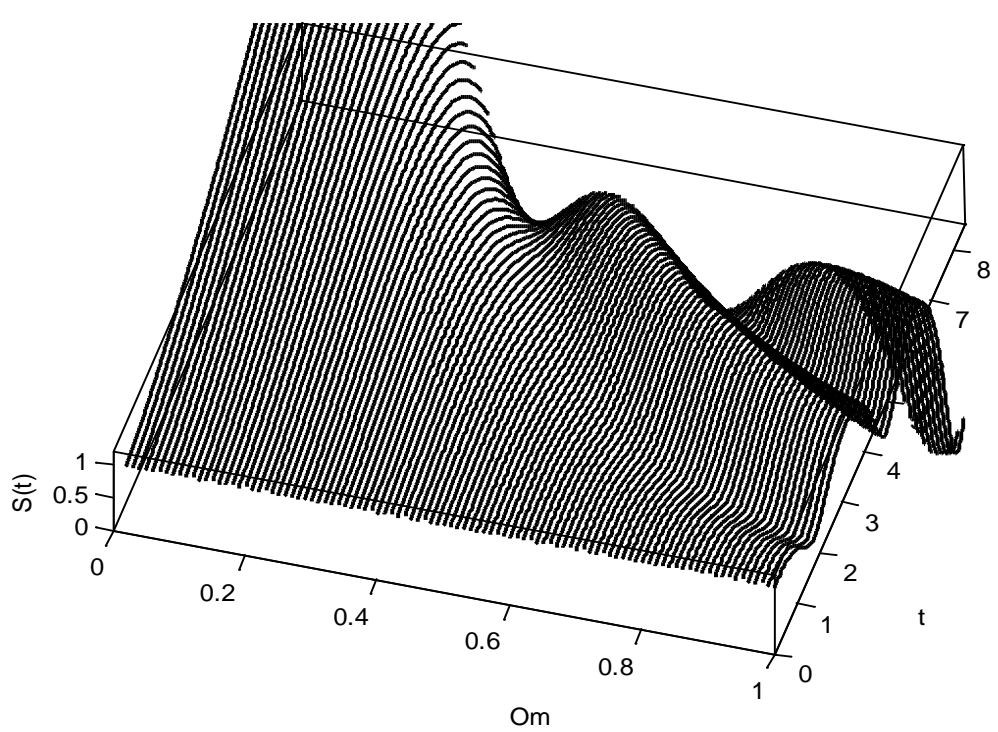

Figure 3. The 3D entropy as function of time and the system frequency for the parameters of Figure 1 with $\gamma=0.1$. The transfer of information between the system and the environment has no periodicity. 
frequency $\Omega$. This plot shows that the Shannon entropy loses its periodicity and decays with the system frequency. Furthermore, this entropy $S_{1}$ grows with time and the damped factor as shown on Figure 4. These results indicate that the damped factor enhances the transfer of information between the system and the environment. Also the fact that the envelope of the curve of Shannon entropy increase implies that this information is losing in time. This traduces the decoherence of the system.

\subsection{System Properties for a Double Gaussian Case}

In this section, we focus our attention on the study of the effects of the double Gaussian approximation function on

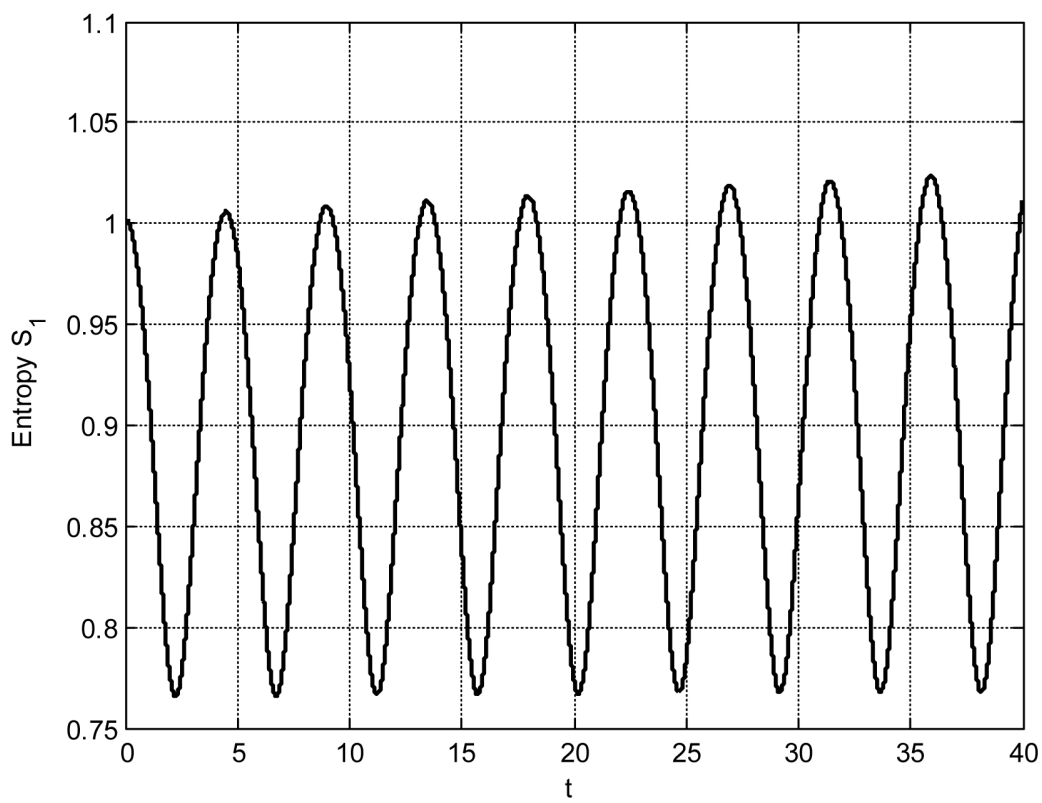

(a)

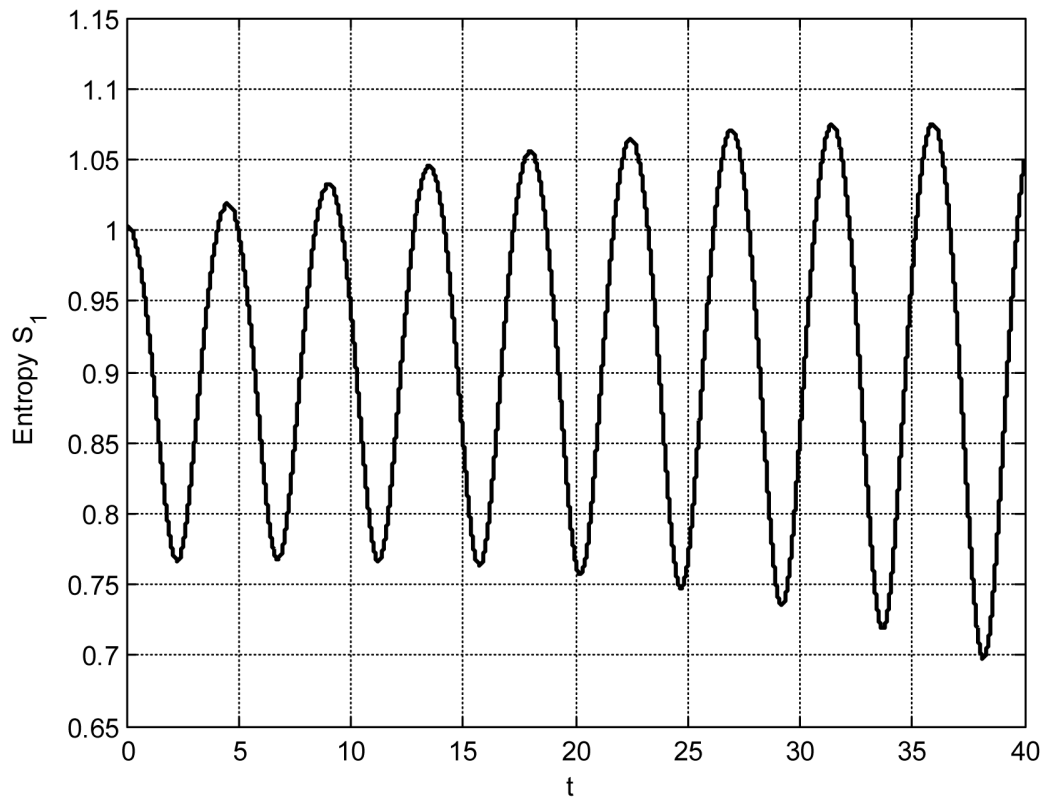

(b)

Figure 4. Behavior of the Shannon entropy $S_{1}(t)$ for several values of the damped factor (a): $\gamma=0.001$ and (b): $\gamma=0.006$. 
the interaction between our damped harmonic oscillator and its environment. For this purpose, the initial state for the double Gaussian wave function is given by [31]

$$
\varphi^{\prime}\left(x_{b}, 0\right)=\left(8 \pi \sigma^{2}\right)^{-1 / 4}\left[1+\exp \left(\frac{-d^{2}}{8 \sigma^{2}}\right)\right]\left\{\exp \left[\frac{-1}{4 \sigma^{2}}\left(x-\frac{d}{2}\right)^{2}\right]+\exp \left[\frac{-1}{4 \sigma^{2}}\left(x+\frac{d}{2}\right)^{2}\right]\right\}
$$

in which $d$ defines the distance between the top of the two successive waves in the double Gaussian state. To appreciate the impact of this new wave packet on the thermodynamic parameters of the system, we seek separately its distribution probability and Shannon entropy.

\subsubsection{Distribution Probability}

The corresponding distribution probability $P^{\prime}$ is obtained by substituting expression (16) into (10). The computation yields the upcoming quantity:

$$
P^{\prime}=\frac{16 \pi A \sigma^{2}}{\sqrt{1-16 a^{2} \sigma^{4}}}\left[\mathrm{e}^{u_{1}}+\mathrm{e}^{u_{2}}\right] \cdot\left[\mathrm{e}^{v_{1}}+\mathrm{e}^{v_{2}}\right]
$$

wherein $\quad u_{1}=\frac{\left(d+4 b \sigma^{2}\right)^{2}}{16 \sigma^{2}\left(1-4 a \sigma^{2}\right)} ; \quad u_{2}=\frac{\left(d-4 b \sigma^{2}\right)^{2}}{16 \sigma^{2}\left(1-4 a \sigma^{2}\right)} ; \quad v_{1}=\frac{\left(d-4 b \sigma^{2}\right)^{2}}{16 \sigma^{2}\left(1+4 a \sigma^{2}\right)} ; \quad v_{2}=\frac{\left(d+4 b \sigma^{2}\right)^{2}}{16 \sigma^{2}\left(1+4 a \sigma^{2}\right)} \quad$ and

$$
A=\frac{\left(8 \pi \sigma^{2}\right)^{-1 / 2} m_{0} \Omega \exp \left(2 \gamma t_{b}-\frac{d^{2}}{8 \sigma^{2}}\right)}{2 \pi \hbar \sin (\Omega t)\left[1+\exp \left(-\frac{d^{2}}{8 \sigma^{2}}\right)\right]} .
$$

One could note that the distribution probability depends not only on time, position and system frequency, but also on the distance separating the two successive peaks of the double Gaussian function. In the limit case $d=0$, we recover the probability (14) that deals with a single Gaussian wave function.

The Spatiotemporal evolution of the probability (17) is plotted on Figure 5. These figures confirm the fact that, the probability grows with the increment of the damped factor $\gamma$ (showing that the information is losing with the increasing of the damping coefficient). Analysis of Figure 5(a) and Figure 1(a) shows that the distance $d$ improves the probability meanwhile the double Gaussian wave function is welcome for the study of the probability of this particle.

\subsubsection{Shannon Entropy}

In this subsection, we investigate the Shannon entropy relates to the double Gaussian wave function for a specific damped harmonic oscillator. Owing to the definition, this entropy is:

$$
S_{2}(t)=-K_{B} \int_{-\infty}^{+\infty} P^{\prime} \ln P^{\prime} \mathrm{d} x
$$

in which the probability $P^{\prime}$ is defined by (17). Hereafter, we explore the influence of each system characteristics on the evolution of this entropy.

First, Figure 6 presents the effects of the distance $d$ on the behavior of the entropy $S_{2}(t)$ for the pure state. From these curves, one observes that the entropy amplitude has an unchanged time behavior for given values of $d$. It appears from these curves that the factor $d$ can be used to control the transfer of information between system and its environment.

Next, we examine the influence of the damped factor $\gamma \neq 0$ on the entropy (18) as shown on Figure 7. These plots confirm the fact that the presence of $\gamma$ induces the decoherence of the system. Comparison of Figure 6(b) and Figure 7(b) shows that the growth of $\gamma$ affects the coherence of the system. At the end, we compare the plot of Figure 7(a) and Figure 7(b), and appreciate the cumulative effects of $d$ and $\gamma$ on the coherence of the system. These graphs let appear that $d$ and $\gamma$ contribute to the decoherence of the system: one $(\gamma)$ increases the magnitude of the entropy while the other $(d)$ reduces the periodicity of the information transferred. 


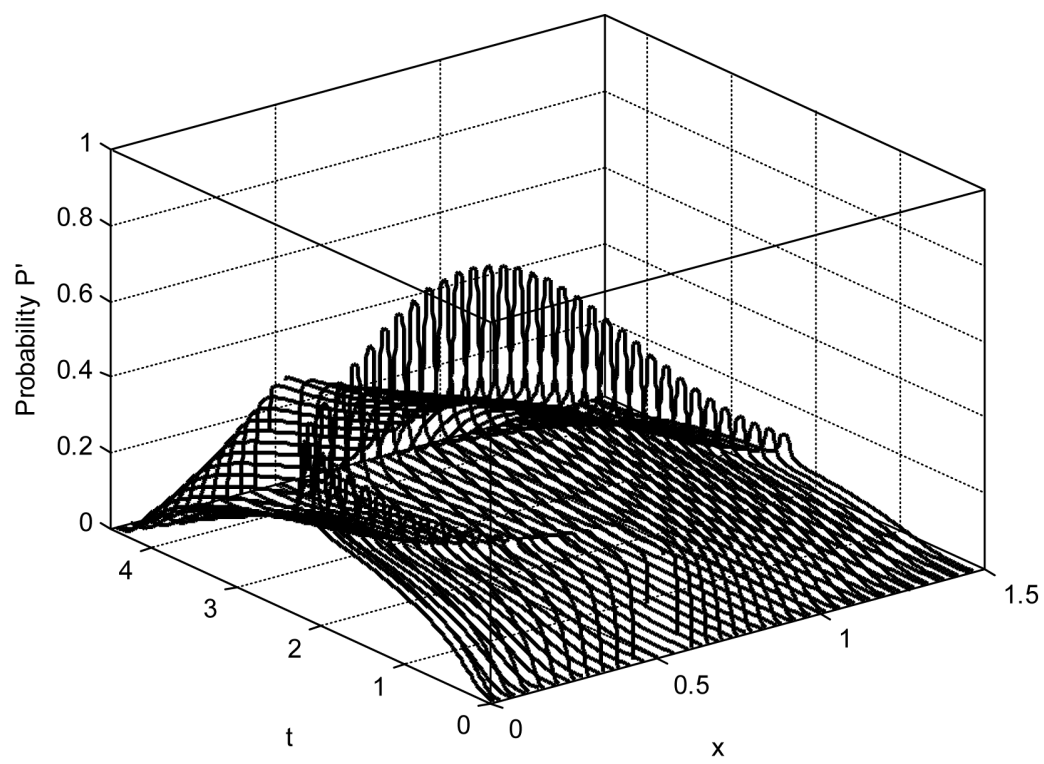

(a)

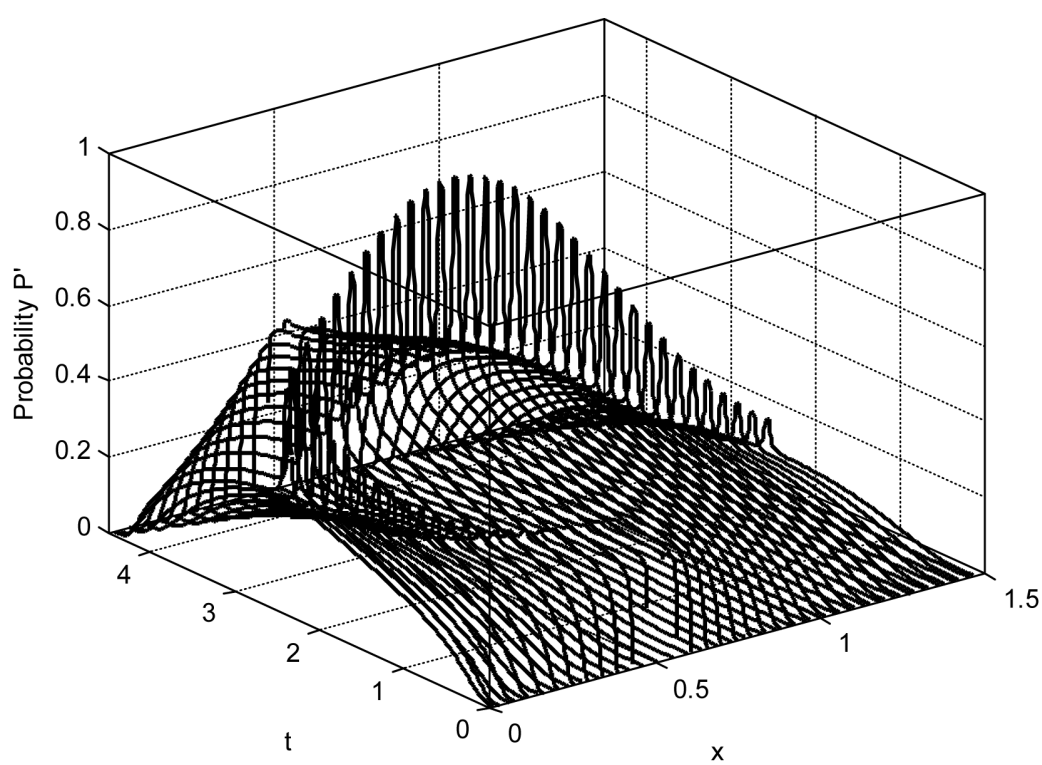

(b)

Figure 5. Distribution probability versus space and time for a double Gaussian wave function with the parameters $\Omega=0.7, m_{0}=1, \hbar=1, d=0.1$ and various values of $\gamma:$ (a): $\gamma=0$ and (b): $\gamma=0.1$.

\section{Conclusions}

In this paper, we have examined the dynamics of the modified damped harmonic oscillator constructed from the Caldirola-Kanaï model and based on the idea of Bateman. For this purpose, the Feynman path integral method has been used to investigate the time dependent probability and the entanglement entropy exploiting the single and double Gaussian initial states. In these two initial states, we have shown that the Shannon entropy decreases with the system frequency and grows with the others parameters (such as time or damped factor). For both cases, we have established that the distribution probability possesses the same behavior. In the specific case of the double Gaussian approximation, we have obtained that the distribution probability and the Shannon's entropy have been improved by the distance between two consecutive peaks of the wave. 


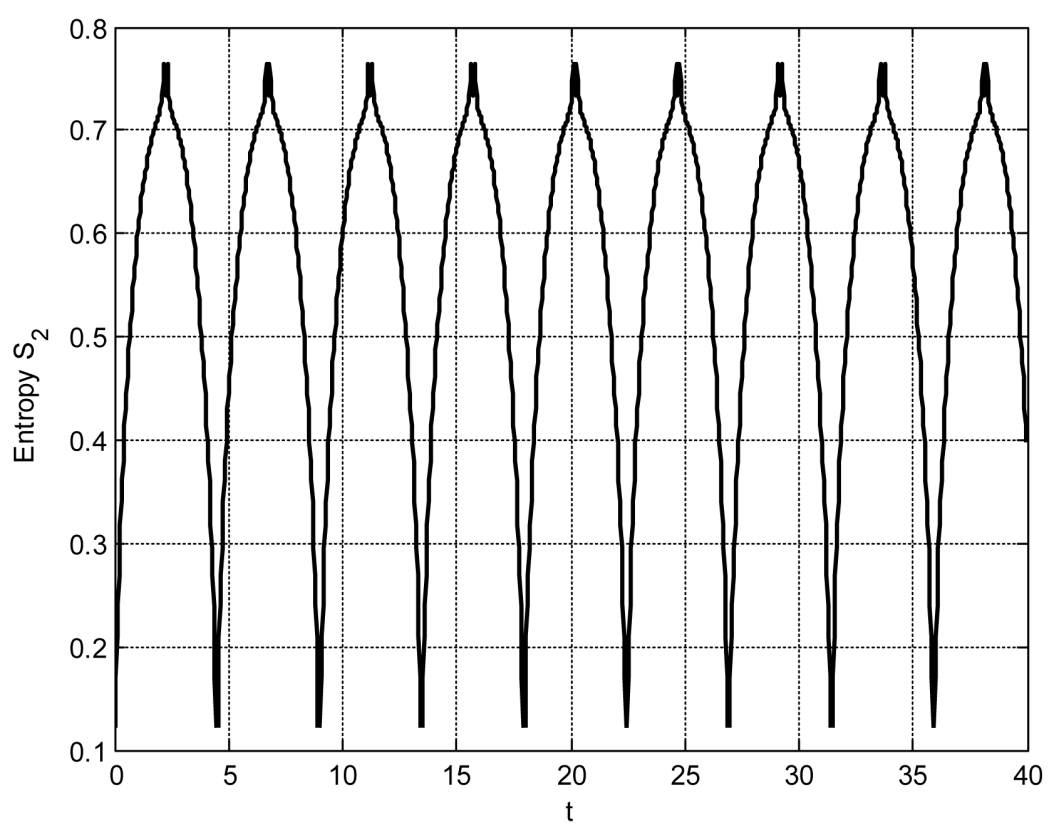

(a)

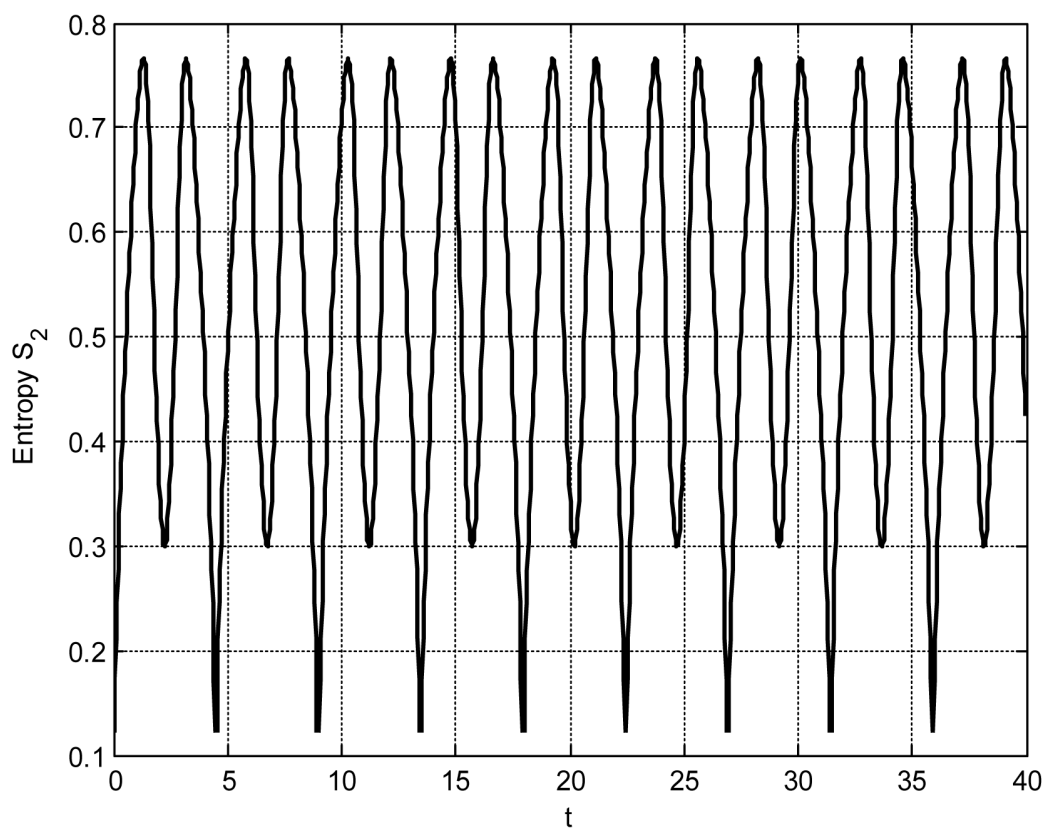

(b)

Figure 6. Shannon entropy $S_{2}(t)$ as function of time for the parameters of Figure

5(a) with several values of $d:(a): d=0.1$ and (b): $d=1.4$.

In the absence of the damped factor, we have recovered the results that link with the system in its pure state. Here, the information is exchanged periodically between the corresponding harmonic oscillator and the environment. For non-zero values of the damped factor, it has appeared that this transfer of information loses its periodicity traducing the loss of information in the system (decoherence phenomena). These results could be of great interest for engineering purposes since it becomes necessary to control the effects of the environment on the evolution of the system in order to reduce its decoherence. This phenomenon of controlling decoherence in an evolving system is essential in the construction of quantum computers that need the use of systems taken in their 


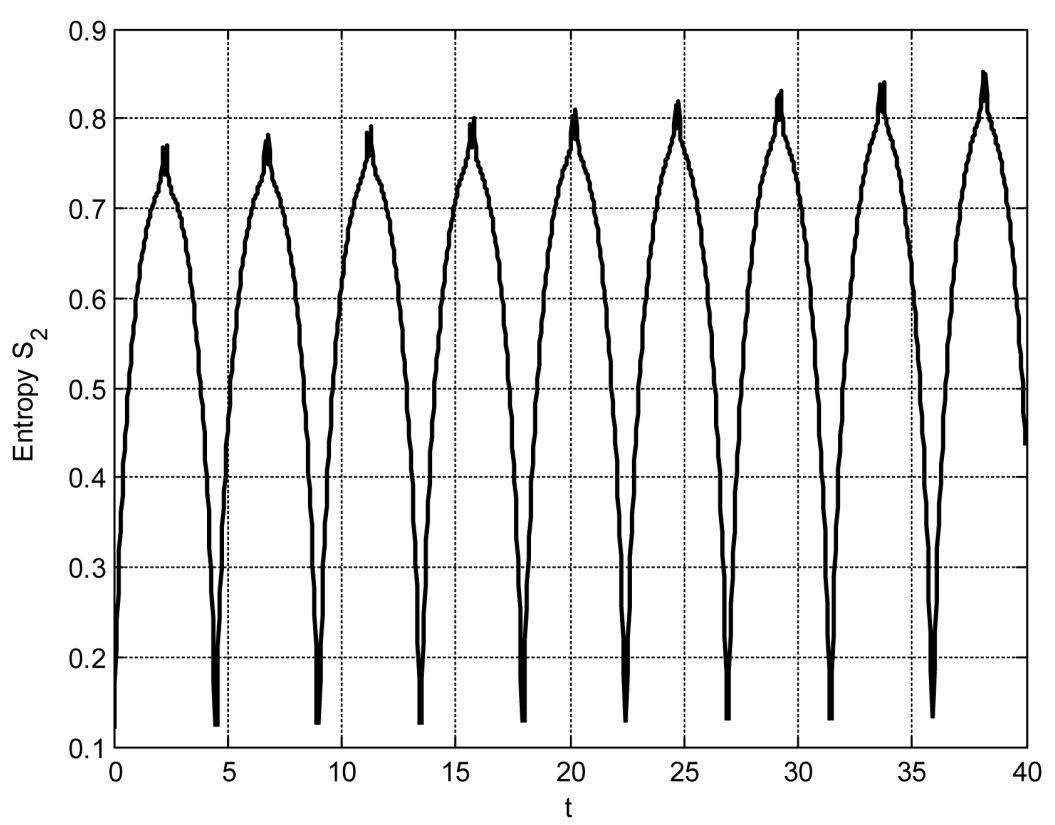

(a)

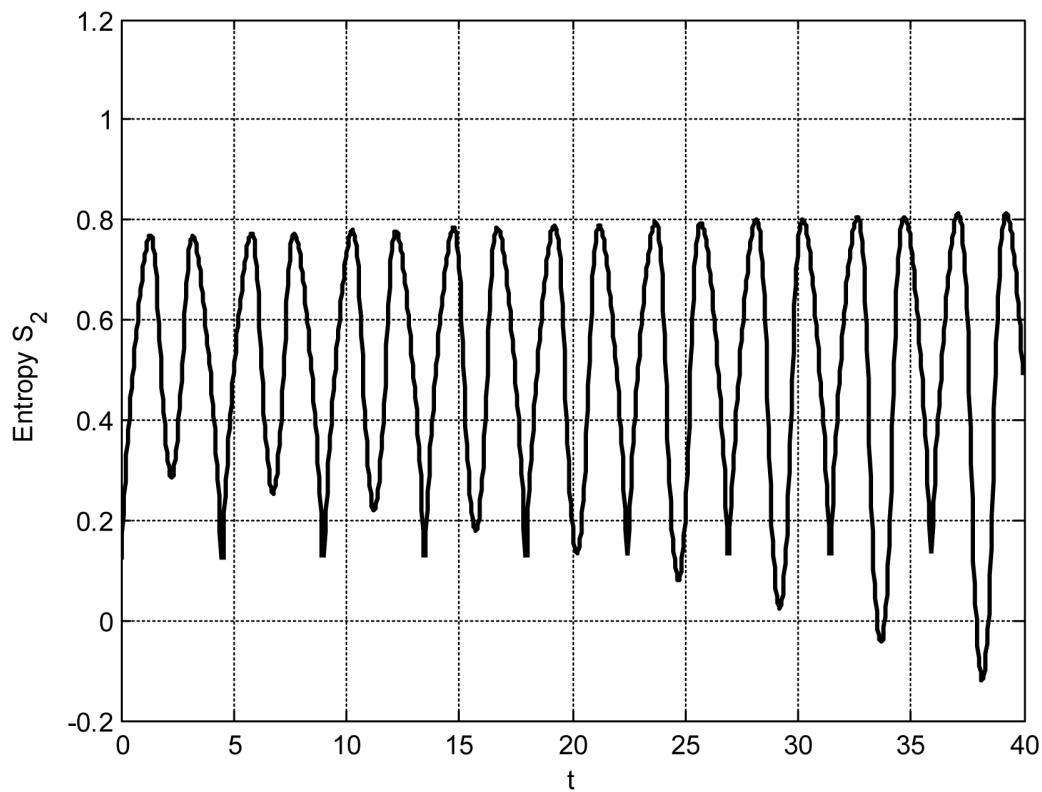

(b)

Figure 7. Temporal evolution of the Shannon entropy for the parameters of Figure 6 with $\gamma=0.003$ and various values of $d:(a): d=0.1$ and (b): $d=1.4$. The factor $d$ can be used to control the decoherence of the system.

different superposition states. Our study has also shown that such aim could be achieved by acting on the damped coefficient, the frequency of oscillation and/or the type of state as control parameter.

\section{References}

[1] Isar, A. (2005) Decoherence in Open Quantum Systems. Romanian Journal of Physics, 50, 147-156.

[2] Walls, D.F. and Millburn, G.J. (1994) Quantum Optics. Springer, Heildelberg.

[3] Scully, M.O. and Zubairy, M.S. (1997) Quantum Optics. Cambridge University Press, Cambridge. 
http://dx.doi.org/10.1017/CBO9780511813993

[4] Sargent III, M., Scully, M.O. and Lamb Jr., W.E. (1974) Laser Physics. Addison-Wesley, Reading.

[5] Nielsen, M.A. and Chuang, I.L. (2000) Quantum Computation and Quantum Information. Cambridge University, Cambridge, UK.

[6] Karlsson, E.B. (1998) Coherence and Decoherence of Positive Particle States in Solids. Physica Scripta, 76, 179. http://dx.doi.org/10.1238/Physica.Topical.076a00179

[7] Chatzidimitriou-Dreismann, C.A., Abdul-Redah, T. and Kolaric, B. (2001) Entanglement of Protons in Organic Molecules: An Attosecond Neutron Scattering Study of C [Bond] H Bond Breaking. Journal of the American Chemical Society, 123, 11945-11951. http://dx.doi.org/10.1021/ja004186d

[8] Chatzidimitriou-Dreismann, C.A., Abdul Redah, T., Streffer, R.M. and Mayers, J. (1997) Anomalous Deep Inelastic Neutron Scattering from Liquid H2O-D2O: Evidence of Nuclear Quantum Entanglement. Physical Review Letters, 79, 2839-2842. http://dx.doi.org/10.1103/PhysRevLett.79.2839

[9] Krzywicki, A. (1993) Coherence and Decoherence in Radiation off Colliding Heavy Ions. Physical Review D, 48, 5190-5195. http://dx.doi.org/10.1103/PhysRevD.48.5190

[10] Zeh, H.D. (1986) Emergence of a Classical Universe from Quantum Gravity and Cosmology. Physics Letters A, 116, 9-12. http://dx.doi.org/10.1016/0375-9601(86)90346-4

[11] Zeh, H.D. (1988) Time in Quantum Gravity. Physics Letters A, 126, 311-317. http://dx.doi.org/10.1016/0375-9601(88)90842-0

[12] Zeh, H.D. (1992) The Physical Basis of the Direction of Time. Springer, Berlin. http://dx.doi.org/10.1007/978-3-662-02759-2

[13] Kiefer, C. (1987) Emergence of a Classical Universe from Quantum Gravity and Cosmology. Classical and Quantum Gravity, 4, 1369-1382. http://dx.doi.org/10.1088/0264-9381/4/5/031

[14] Halliwell, J. (1989) Decoherence in Quantum Cosmology. Physical Review D, 39, 2912-2923. http://dx.doi.org/10.1103/PhysRevD.39.2912

[15] Barvinsky, A.O. and Kamenshchik, A.Y. (1990) Preferred Basis in the Many-Worlds Interpretation of Quantum Mechanics and Quantum Cosmology. Classical and Quantum Gravity, 7, 2285-2293. http://dx.doi.org/10.1088/0264-9381/7/12/010

[16] Barvinsky, A.O. and Kamenshchik, A.Y. (1995) Preferred Basis in Quantum Theory and the Problem of Classicalizationof the Quantum Universe. Physical Review D, 52, 743-757. http://dx.doi.org/10.1103/PhysRevD.52.743

[17] Brandenberger, R., Laflamme, R. and Mijic, M. (1990) Modern Physics Letters A, 5, 2311.

[18] Paz, J.-P. and Sinha, S. (1991) Decoherence and Back Reaction: The Origin of the Semiclassical Einstein Equations. Physical Review D, 44, 1038-1049. http://dx.doi.org/10.1103/PhysRevD.44.1038

[19] Paz, J.-P. and Sinha, S. (1992) Decoherence and Back Reaction in Quantum Cosmology: Multidimensional Minisuperspace Examples. Physical Review D, 45, 2823-2842. http://dx.doi.org/10.1103/PhysRevD.45.2823

[20] Castagnino, M.A., Gangui, A., Mazzitelli, F.D. and Tkachev, I.I. (1993) Third Quantization, Decoherence and the Interpretation of Quantum Gravity in Mini-Super-Space. Classical and Quantum Gravity, 10, 2495-2504. http://dx.doi.org/10.1088/0264-9381/10/12/008

[21] Kefer, C. and Zeh, H.D. (1995) Arrow of Time in a Recollapsing Quantum Universe. Physical Review D, 51, 41454153. http://dx.doi.org/10.1103/PhysRevD.51.4145

[22] Mensky, M.B. and Novikov, I.D. (1996) Decoherence Caused by Topology in a Time-Machine Spacetime. International Journal of Modern Physics D, 5, 1-27. http://dx.doi.org/10.1142/S0218271896000023

[23] Giulini, D., Joos, E., Kiefer, C., Kupsch, J., Stamatescu, I.O. and Zeh, H.D. (1996) Decoherence and the Appearance of a Classical World in Quantum Theory. Springer, Berlin. http://dx.doi.org/10.1007/978-3-662-03263-3

[24] Paz, J.P. and Zurek, W.H. (2001) In Coherent Atomic Matter Waves. In: Kaiser, R., Westbrook, C. and David F., Eds., Les Houches Session LXXII, Springer, Berlin, 533-614.

[25] Zurek, W.H. (2003) Decoherence, Einselection, and the Quantum Origins of the Classical. Reviews of Modern Physics, 75, 715-775. http://dx.doi.org/10.1103/RevModPhys.75.715

[26] Morikawa, M. (1990) Quantum Decoherence and Classical Correlation in Quantum Mechanics. Physical Review D, 42, 2929-2932. http://dx.doi.org/10.1103/PhysRevD.42.2929

[27] Habib, S. and Laflamme, R. (1990) Wigner Function and Decoherence in Quantum Cosmology. Physical Review D, 42, 4056-4065. http://dx.doi.org/10.1103/PhysRevD.42.4056

[28] Alicki, R. (2004) Pure Decoherence in Quantum Systems. Open Systems \& Information Dynamics (OSID), $11,53-61$. 
http://dx.doi.org/10.1023/B:OPSY.0000024755.58888.ac

[29] Joos, E., Zeh, H.D., Kiefer, C., Giulini, D., Kupsch, J. and Stamatescu, I.O. (2003) Decoherence and the Appearance of a Classical World in Quantum Theory. Springer, Berlin. http://dx.doi.org/10.1007/978-3-662-05328-7

[30] Gamble, J.K. and Lindner, J.F. (2009) Demystifying Decoherence and the Master Equation of Quantum Brownian Motion. American Journal of Physics, 77, 244. http://dx.doi.org/10.1119/1.3043847

[31] Zuo, J. and O’Connell, R.F. (2004) Effect of an External Field on Decoherence: Part II. Journal of Modern Optics, 51, 821-832. http://dx.doi.org/10.1080/09500340408233599

[32] Ozcan, O., Akturk, E. and Sever, R. (2008) Time Dependence of Joint Entropy of Oscillating Quantum Systems. International Journal of Theoretical Physics, 47, 3207-3218. http://dx.doi.org/10.1007/s10773-008-9756-4

[33] Isar, A. (2007) Quantum Decoherence of a Damped Harmonic Oscillator. Optics and Spectroscopy, 103, 252-257. http://dx.doi.org/10.1134/S0030400X07080140

[34] Lindblad, G. (1976) Brownian Motion of a Quantum Harmonic Oscillator. Reports on Mathematical Physics, 10, 393406. http://dx.doi.org/10.1016/0034-4877(76)90029-X

[35] Sandulescu, A. and Scutaru, H. (1987) Open Quantum Systems and the Damping of Collective Models in Deep Inelastic Collisions. Annals of Physics, 173, 277-317. http://dx.doi.org/10.1016/0003-4916(87)90162-X

[36] Elran, Y. and Brumer, P. (2004) Decoherence in an Inharmonic Oscillator Coupled to a Thermal Environment: A Semi-Classical Forward-Backward Approach. The Journal of Chemical Physics, 121, 2673. http://dx.doi.org/10.1063/1.1766009

[37] Chruscinski, D. and Jurkowski, J. (2010) Memory in a Nonlocally Damped Oscillator. In: Accardi, L., Freudenberg, W. and Ohya, M., Eds., Quantum Bio-Informatics III, Tokyo University of Science, Tokyo, 155-166. http://dx.doi.org/10.1142/9789814304061_0014

[38] Bateman, H. (1931) On Dissipative Systems and Related Variational Principles. Physical Review, 38, 815-819. http://dx.doi.org/10.1103/PhysRev.38.815

[39] Caldirola, P. (1941) Quantum Analysis of Modified Caldirola-Kanai Oscillator Model for Electromagnetic Fieldsin Time-Varying. IL Nuovo Cimento, 18, 393-400. http://dx.doi.org/10.1007/BF02960144

[40] Feynman, R.P. (1948) Space-Time Approach to Non-Relativistic Quantum Mechanics. Reviews of Modern Physics, 20, 367-387. http://dx.doi.org/10.1103/RevModPhys.20.367

[41] Fai, L.C., Fomethe, A., Mborong, V.B., Fotue, A.J., Domngang, S., Issofa, N. and Tchoffo, M. (2008) Polaron State Screening by Plasmons in a Spherical Nanocrystal. Journal of Low Temperature Physics, 152, 71-87. http://dx.doi.org/10.1007/s10909-008-9803-9

[42] Um, C.I., Yeon, K.H. and George, T.F. (2002) The Quantum Damped Harmonic Oscillator. Physics Reports, 362, 63192. http://dx.doi.org/10.1016/S0370-1573(01)00077-1

[43] Um, C.I., Yeon, K.H. and Kahng, W.H. (1987) The Quantum Damped Driven Harmonic Oscillator. Journal of Physics A: Mathematical and General, 20, 611-626. http://dx.doi.org/10.1088/0305-4470/20/3/024

[44] Feynman, R.P. and Hibbs, A.R. (1965) Quantum Mechanics and Path Integrals. McGraw-Hill, New York. 
Scientific Research Publishing (SCIRP) is one of the largest Open Access journal publishers. It is currently publishing more than 200 open access, online, peer-reviewed journals covering a wide range of academic disciplines. SCIRP serves the worldwide academic communities and contributes to the progress and application of science with its publication.

Other selected journals from SCIRP are listed as below. Submit your manuscript to us via either submit@scirp.org or Online Submission Portal.
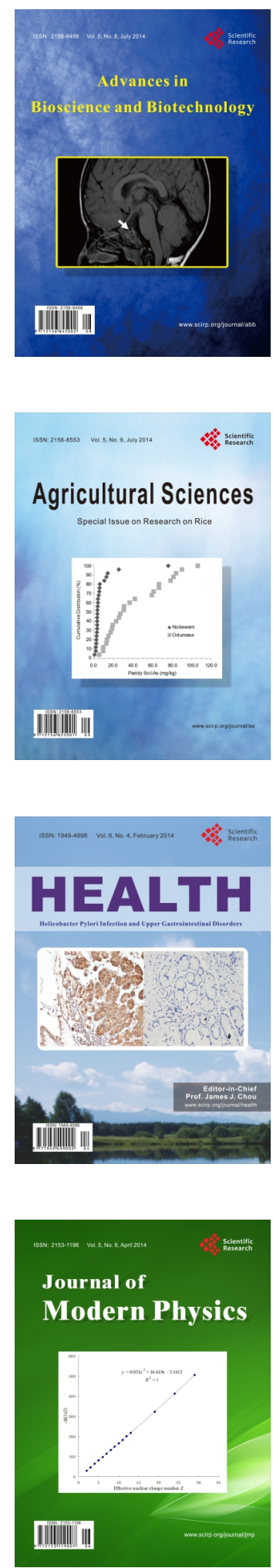
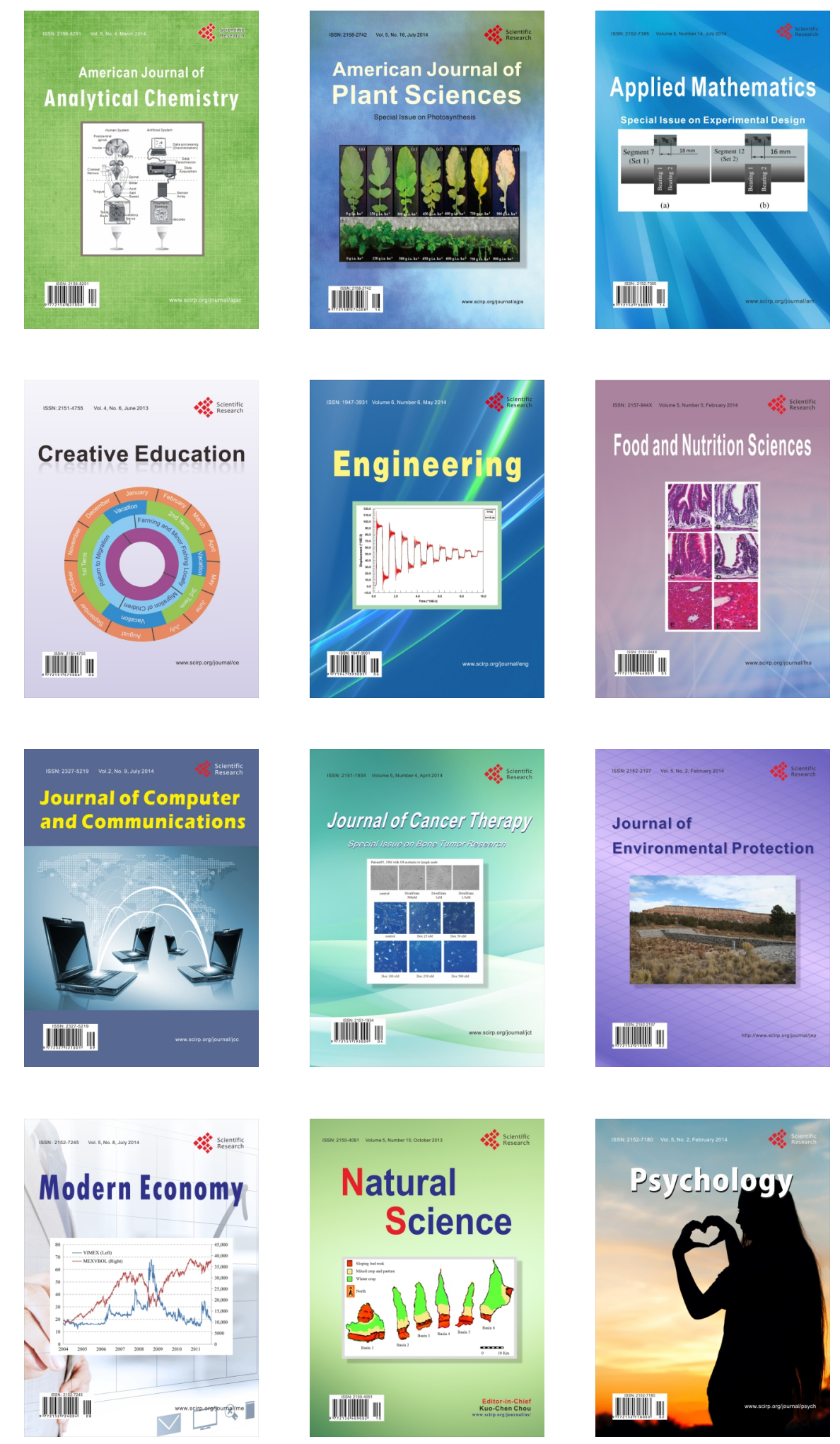